\title{
Influence of the electronic structure on tunneling through ferroelectric insulators: Application to $\mathrm{BaTiO}_{3}$ and $\mathrm{PbTiO3}_{3}$
}

\author{
D. Wortmann and S. Blügel \\ Peter Grünberg Institut and Institute for Advanced Simulation, Forschungszentrum Jülich and JARA, DE-52425 Jülich, Germany
}

(Received 30 July 2010; revised manuscript received 3 March 2011; published 15 April 2011)

\begin{abstract}
Electronic tunneling through ferroelectric insulators is considered to be a key ingredient of future oxide electronics. We investigate the role of the electronic band structure of the decaying electronic states in the band gap by first discussing the expected behavior of tunneling in the effective mass model. We demonstrate that, even for the simple prototype ferroelectric oxides in the perovskite structures $\mathrm{PbTiO}_{3}$ and $\mathrm{BaTiO}_{3}$, the basic assumption of the effective mass model is not appropriate, and that the correct interpretation of tunneling in these materials requires a material-specific description of the evanescent states as provided by the complex band structure.
\end{abstract}

DOI: 10.1103/PhysRevB.83.155114

PACS number(s): 73.20.At, 73.40.Gk, 77.84.Cg

\section{INTRODUCTION}

The possibility to change the electronic tunneling transmission through a ferroelectric tunneling barrier by switching its polarization state is an intriguing concept for new devices. ${ }^{1-3}$ Experiments using ferroelectrics in tunnel junctions or in piezoresponse force microscopy have already been established demonstrating the basic functionality. ${ }^{4-8}$ The additional degree of freedom provided by the ferroelectric state of the barrier can also be exploited in magnetic tunnel junctions, opening the path to a 2-bit memory cell. ${ }^{4}$ While the basic ideas and the corresponding theoretical description were established already, ${ }^{1,9,10}$ actual theoretical investigations of realistic tunneling setups on the basis of an ab initio description of the material are still rare. ${ }^{11,12}$ These calculations are rather difficult to perform and even more difficult to relate to experimental findings due to the enormous number of possible atomic realizations of an actual tunnel junction with a ferroelectric barrier. As a consequence, most predictions of the tunneling electroresistance effect in these setups are based on model calculations, $, 2,9,10,13$ usually assuming a parabolic dispersion of the bands. In this paper, we focus on improving the description of this specific aspect of the problem: the electronic property of the bulk barrier material. Very recently, a similar approach was published by Hinsche et al. ${ }^{14}$ combining a simple screening model for the electrodes with details of the electronic structure of the barrier to investigate the dependence of the tunneling on the polarization of the barrier material.

Disregarding any effects due to possible disorder, defects, or dislocations, different physical effects can be imagined that will lead to a modification of the tunneling through a perfect ferroelectric barrier when switching its polarization ${ }^{9}$ : (i) As all ferroelectrics are piezoelectrics, the change in the polarization will lead to a structural modification that can influence the tunneling. ${ }^{9,15,16}$ In particular, structural modifications that lead to a change in the thickness of the barrier can be expected to have a significant impact on the tunneling current. While these effects may be exploited for some applications in sensors, ${ }^{17}$ they could also impose problems in highly integrated devices due to the strain that they can exert on their environment. (ii) The modification of the interface between the ferroelectric barrier and the metallic leads. The change of the polarization will strongly affect these interfaces, inducing large screening charges and modifying the chemical binding. These modifications change the matching of the electronic wave functions at the interface and, hence, will modify the tunneling probability of electrons. In general, this purely electronic effect can be expected to be quite substantial as the tunneling current is typically carried by only a few states with energies close to the Fermi level, and any modification of these states directly will be reflected in the electronic transmission. (iii) As a third effect, the switching of the polarization can change the electronic properties of the tunneling barrier itself. In particular, the difference in band alignment at the interfaces will manifest itself in a change of the local potential within the tunnel barrier, which will influence the decay of the wave function and, thus, the tunneling probability.

We will first summarize the basic predictions of a simple model of tunneling with focus on the relation between the decay of the wave function in the barrier and the height of the barrier. We will then discuss two prototype ferroelectric barrier materials from the group of perovskite transition-metal-oxide ferroelectrics: $\mathrm{BaTiO}_{3}$ and $\mathrm{PbTiO}_{3}$. These materials have been investigated in detail both theoretically and experimentally before. We will, hence, concentrate only on the description of the evanescent states relevant for tunneling. We will show that, for these materials, the simple interpretation of tunneling in terms of a free-electron picture breaks down. To demonstrate this breakdown, we calculate the decay constant $\kappa$ of electrons tunneling through an insulating barrier from the realistic electronic band structure. In the simple effective mass approximation, this decay constant is linked to the height of the potential barrier $V_{0}$ relative to the energy of the tunneling electron and to the effective mass $m$ as

$$
\kappa=\frac{1}{\hbar} \sqrt{2 m V_{0}} .
$$

This assumption is underlying many theoretical predictions, but has only little applicability to transition-metal oxides.

\section{SIMPLE TUNNELING THEORY}

Electronic tunneling can be treated on various levels. If one considers the small-bias regime, the problem can 
be investigated using the theory of linear response in the Kubo formalism. While this is a very general approach, in practice, one often restricts the interpretation to the case of noninteracting electrons being scattered at the barrier potential. In this case, tunneling is described by the famous Landauer equation $^{18}$ in which the key quantity is the transmission probability of electrons through the barrier. A well-known trivial example of this formalism yields the conductance across a simple one-dimensional potential barrier of constant height $V_{0}$ sandwiched between two free-electron gases. Here, one obtains for the tunneling conductance

$$
\begin{aligned}
\Gamma & =\frac{e^{2}}{h} \frac{(4 \kappa k)^{2}}{\left(k^{2}+\kappa^{2}\right)^{2}\left(1-e^{-2 \kappa d}\right)^{2}+(4 \kappa k)^{2} e^{-2 \kappa d}} e^{-2 \kappa d} \\
& \approx \frac{e^{2}}{h} \frac{(4 \kappa k)^{2}}{\left(k^{2}+\kappa^{2}\right)^{2}} e^{-2 \kappa d} .
\end{aligned}
$$

In this equation, the exponential factor $e^{-2 \kappa d}$ describes the exponential decay of the wave function with a decay constant of $\kappa=\frac{1}{\hbar} \sqrt{2 m V_{0}}$ within the barrier of thickness $d$. The interface matching of the wave functions is controlled by the values of $\kappa$ and the $k$ value describing the free-electron states in the leads. For thick barriers, the backscattering contribution corresponding to the exponentially small terms in the denominator can be neglected, and one obtains the approximate form in the second line in which the conductance is simply proportional to the exponential decay factor.

In the case of a ferroelectric barrier material, the electric polarization within the barrier can lead to a nonconstant barrier height. Neglecting all local variations of the polarization within the barrier, one would obtain a barrier potential that changes linearly within the barrier with some finite slope. Hence, one aims at generalizing the result of Eq. (2) by simply assuming that the decay constant $\kappa$ now varies within the barrier. The simplest generalization of Eq. (2), which can also be interpreted as the well-known Wenzel-Kramers-Brillouin (WKB) approximation for the tunneling of electrons, is given by

$$
\Gamma=C e^{-2 \int_{0}^{d} \kappa(z) d z}=C e^{-2 \bar{\kappa} d},
$$

where $\bar{\kappa}$ is simply the averaged decay constant across the barrier and the constant $C$ contains all further interface details.

When switching the polarization direction, the barrier potential will change. This change of the barrier will be actually induced by the modification of the charges at the interfaces. The polarization manifests itself by an effective interface charge, which will, in turn, be screened by the metal leads. In general, this screening will be incomplete and a remaining depolarizing field corresponding to a barrier potential of finite slope is expected. We refer to Ref. 10 for a more detailed discussion of the screening at the interfaces.

Due to time-inversion symmetry, the change of the barrier potential can only lead to a change in tunneling conductance if the total tunnel junction is not symmetric, i.e., if the barrier after switching the polarization is not simply the mirror image of the unswitched barrier. For small-bias voltages, this requires that the two interfaces between the leads and the ferroelectric barrier are different, so that the induced screening charges within the metallic leads and/or the details of the chemical and atomic arrangement are different. ${ }^{9}$ Similarly, at larger applied voltage, the additional bias potential also breaks the symmetry between the two polarization states. In the resulting asymmetric case, the shape of the potential barrier will be different for the two directions of the polarization leading to a tunneling electroresistance (TER) given by

$$
\mathrm{TER}=\frac{\Gamma_{\leftarrow}-\Gamma_{\rightarrow}}{\Gamma_{\leftarrow}+\Gamma_{\rightarrow}} \approx \tanh d\left(\overline{\kappa_{\rightarrow}}-\overline{\kappa_{\leftarrow}}\right) .
$$

Here we again assumed that the matching at the interface is the same for both polarization directions, i.e., the factor $C$ in Eq. (3) is the same for the conductance in both directions of polarization $\Gamma_{\leftarrow}$ and $\Gamma_{\rightarrow}$. In the effective mass model, one can easily evaluate the average decay constant and the resulting TER. Assuming that the barrier height is changing linearly across the barrier, the decay constant is given by $\kappa(z)=\frac{1}{\hbar} \sqrt{\frac{2 m}{d}\left[V_{1}(d-z)+V_{2} z\right]}$, where $V_{1}$ and $V_{2}$ are the barrier heights at both ends of the tunneling barrier (i.e., at $z=0$ and $z=d$ ) (see Fig. 1). The resulting averaged decay constant is then

$$
\bar{\kappa}=\frac{2}{3} \frac{1}{\hbar} \frac{\sqrt{2 m}\left(V_{1}^{3 / 2}-V_{2}^{3 / 2}\right)}{\left(V_{1}-V_{2}\right)}
$$

and the tunneling electroresistance ratio can be expressed in terms of the barrier heights. Introducing the averaged barrier height $\bar{V}$ and setting $V_{1}=\bar{V}+\frac{1}{2} \Delta V$ and $V_{2}=\bar{V}-\frac{1}{2} \Delta V$, one obtains, up to order of $\Delta V^{4}$,

$$
\bar{\kappa} \approx \frac{1}{\hbar} \sqrt{2 m \bar{V}}-\frac{1}{\hbar} \sqrt{2 m} \frac{1}{6 \bar{V}^{3 / 2}} \Delta V^{2} .
$$

This simple analysis demonstrates a few basic features of the TER due to the change of the barrier potential:

(1) A change in the average potential height $\bar{V}$ will lead to a variation of the tunneling transmission. While the effect of this change of average potential can be expected to be strong, it will usually be accompanied by a change in energy of the system, i.e., the system will have a preferred orientation of the polarization, which might not be a desirable effect for many application scenarios.

(2) A change of slope of the barrier, i.e., a modification of $\Delta V$, will result in a TER effect. This is due to the nonlinearity of the decay constant as a function of barrier height and the

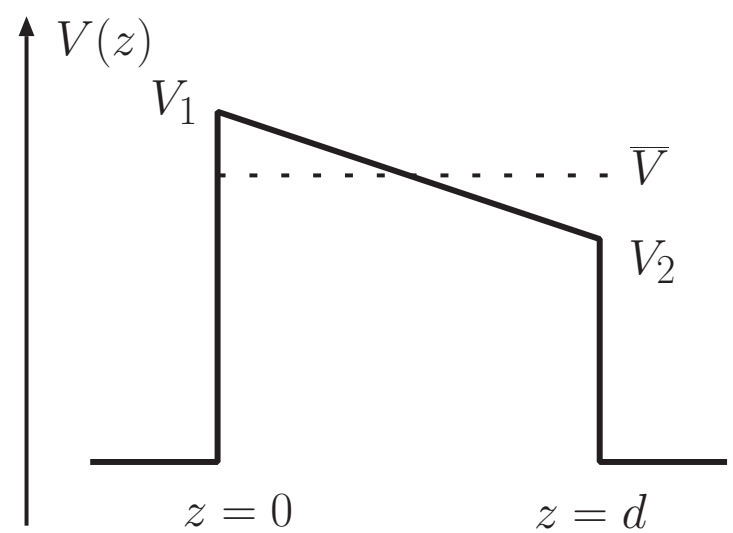

FIG. 1. Simplest model of a potential barrier with a ferroelectric barrier. The barrier height is measured with respect to the energy of the propagating electrons in the leads. 
effect will depend on the average barrier height. A variation close to the band edge, i.e., with a small average potential $\bar{V}$ will be more significant as one can expect from the parabolic dispersion of the decaying band.

(3) As the TER is due to the difference of the electronic structure in the barrier, it scales with the barrier thickness [see Eq. (4)]. This is a fundamental difference to the TMR in which the spin polarization of the leads and the interface are fundamental for the effect. One should also note here that the TER does not directly depend on a residual field in the barrier. Such a field will decay with increasing thickness. As averaged decay constants $\bar{\kappa}_{\leftarrow \rightarrow}$ depend on the potential alignment at the interfaces, they can be expected to be largely independent of the barrier thickness $d$, with a TER rising $\propto \tanh d \Delta \bar{\kappa}$.

At this point, one should clarify some of the simplifications introduced by our model. Most seriously, we assume the decay of the wave function to be a function of the barrier height only. In reality, the barrier material will of course respond to the fields in the barrier and, e.g., the magnitude of the polarization will change upon reversal. While these effects can be the source of a TER effect, they are not within the scope of our paper. In fact, we do not treat the polarization of the material explicitly except for the fact that we consider it as the source of the potential variation in the barrier. However, one should not confuse this potential variation with the polarization of the material itself. As stressed before, the barrier potential will be determined by the interface charges.

\section{COMPLEX BAND STRUCTURE OF FERROELECTRICS}

In a realistic material, the decay constant usually does not follow the simple square-root form suggested by the effective mass model. In a generalization of the usual band-structure theory for the propagating Bloch states, evanescent states in a material can be described by states with a complex $k$ value $k=q+i \kappa$, where $q$ denotes the real part of the Bloch vector and the imaginary part is the decay constant $\kappa$. The theory of this so-called complex band structure, i.e., of the set of real energies $\epsilon(q+i \kappa)$, was developed by Heine. ${ }^{19}$ Several approaches to obtain the complex band structure from $a b$ initio density functional theory (DFT) calculations have been developed (e.g., Refs. 20-22). In such calculations, one usually aims at solving the inverse problem of the standard DFT, i.e., instead of fixing the Bloch vector and solving for the energy eigenstates, one fixes the energy and solves for the real and complex Bloch vectors. In the following, we will present results of such complex band-structure calculations using our all-electron Green's function embedding method ${ }^{23}$ implemented into the full-potential augmented-plane wave FLEUR code. ${ }^{24}$ Our calculations were performed using the generalized gradient approximation (GGA) $\left[\right.$ for $\mathrm{BaTiO}_{3}$ (Ref. 25) and for $\mathrm{PbTiO}_{3}$ (Ref. 26)]. While this treatment is not expected to yield correct absolute values of, e.g., the band gaps or the effective masses, the general trends as well as the connectivity of the band structure can be trusted. In the following, we will dwell on these features.

As the first example of a possible ferroelectric barrier material, we consider $\mathrm{BaTiO}_{3}$, one of the simplest and best studied ferroelectric perovskites. It exhibits a large electric polarization even at room temperature and, hence, might be an ideal candidate for a ferroelectric tunnel-barrier material. The ferroelectric phase is obtained starting from the cubic perovskite structure by a tetragonal distortion of the unit cell and a buckling in the $\mathrm{BaO}$ and $\mathrm{TiO}_{2}$ layers.

To model the experimental growth on a substrate, we fixed the in-plane lattice constant to that of $\mathrm{a} \mathrm{SrTiO}_{3}$ substrate (with the corresponding theoretical GGA lattice constant of $3.95 \AA$ ) and relaxed the structure in the out-of-plane direction, which resulted in a unit-cell height of $4.13 \AA$. The $\mathrm{Ti}$ atoms are displaced by $-0.05 \AA$ from the center of the tetragonal cell spanned by the $\mathrm{Ba}$ atoms at the corners of the unit cell, while the $\mathrm{O}$ atoms in the $\mathrm{Ba}$ layer are shifted by $0.24 \AA$ and the $\mathrm{O}$ atoms of the Ti layer by $0.15 \AA$. The very same structure has been presented in Ref. 27 and its ferroelectric polarization has been calculated to be $49 \mu \mathrm{C} / \mathrm{cm}^{2}$.

$\mathrm{BaTiO}_{3}$ is an indirect gap insulator with the minimum of the conduction band at the $\Gamma$ point and the maximum of the valence band at the $R$ point. The smallest direct gap of $2.4 \mathrm{eV}$ can be found at the $\Gamma$ point. Typical for the perovskite structure are the very flat bands in some parts of the Brillouin zone, in particular, along the $\Gamma-X$ line. These are a consequence of the very small overlap that states have in some crystal directions as, for example, the small overlap of $d_{x^{2}-y^{2}}$ in the $z$ direction. The tetragonal distortion in the ferroelectric phase modifies the band structure around the gap significantly. In particular, most band degeneracies found at the high-symmetry points of the Brillouin zone for the cubic phase are lifted.

Figure 2 shows the complex band structure of $\mathrm{BaTiO}_{3}$ corresponding to states propagating in the [100] direction parallel to the polarization axis. In a (100)-oriented tunnel junction, electronic states with normal incidence correspond to the uppermost panel $\left(k_{\|}=0\right)$, while the other two panels show the bands relevant for nonvanishing momentum in the plane of the interface. Most interestingly, the band edges are formed by states with high effective mass only. The bottom of the conduction band is realized by the flat band of $\Delta_{2}$ symmetry at the $\Gamma$ point, which can be seen in the top panel of Fig. 2, and the valence-band maximum is found at the $R$ point by the maximum of a flat band of $T_{1^{\prime}}$ symmetry. As all these states at the band edges have little dispersion, the corresponding evanescent states very quickly acquire a large imaginary $k_{\perp}$ value so that these states will not be relevant for tunneling, except at energies very close to the band edges. For $\vec{k}_{\|}=0$, the most slowly decaying state over (nearly) the whole band gap is given by the evanescent band starting at around $-1 \mathrm{eV}$. Similar bands can also be identified at the other $\vec{k}_{\|}$ values shown in Fig. 2. Over the energy range of the band gap, these bands show little variation of $\kappa=\operatorname{Im}\left(k_{\perp}\right)$, i.e., they form roughly vertical lines in Fig. 2. The surprising consequence is the similar attenuation of tunneling electrons with different energies, i.e., all states will decay similarly fast. In the effective mass model, this would correspond to potential barriers of equal height.

This finding of roughly constant decay for different $\vec{k}_{\|}$and almost all energies within the band gap is confirmed by the calculation of the minimal decay constant for all $\vec{k}_{\|}$as plotted in Fig. 3. One should note that the color scale spans only a small range in this plot. This reflects the fact that the decay 


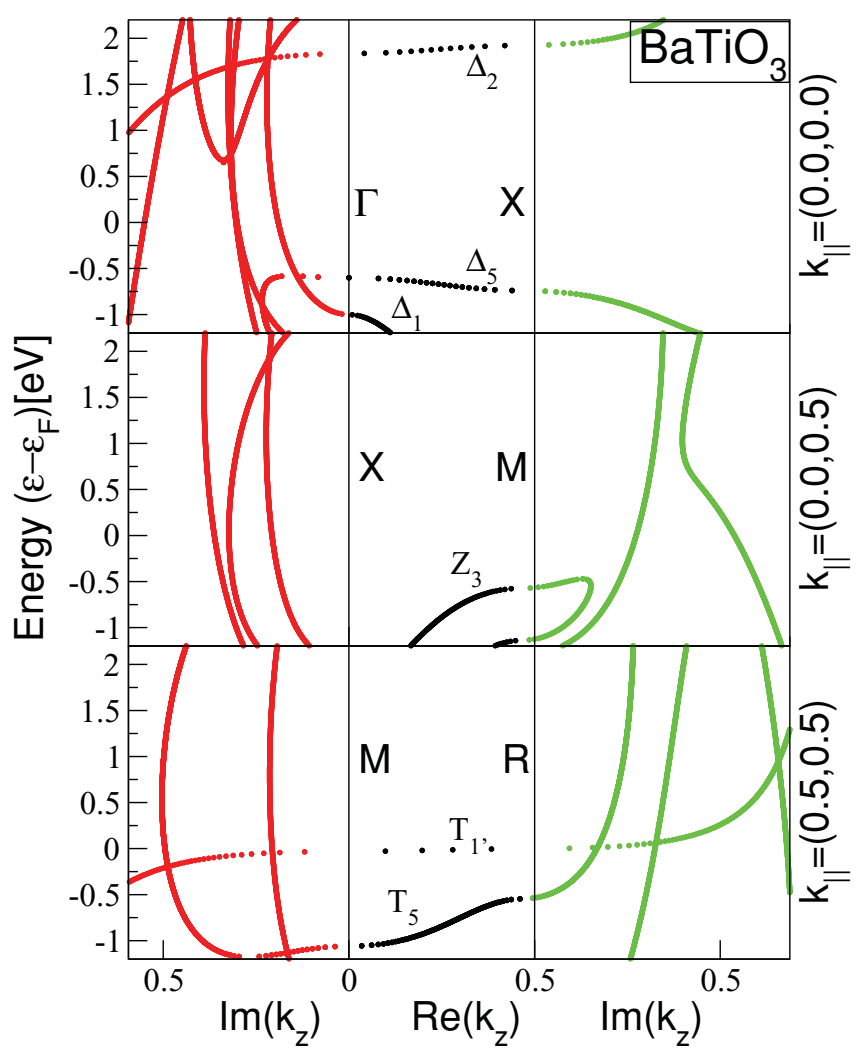

FIG. 2. (Color online) Complex band structure of $\mathrm{BaTiO}_{3}$. Plotted are the energies of the states in $\mathrm{eV}$ with respect to the Fermi level $\varepsilon_{F}$ defined by the highest occupied state as a function of $k_{z}$ given in internal units (i.u. $=2 \pi / 3.95 \AA$ ). The three panels show the bands for different $k_{\|}$corresponding to different angles of incident electrons on the (001) surface. Black dots (central column) represent usual Bloch states with $\Im\left(k_{z}\right)=0$, red dots (left column) evanescent states with $\Re\left(k_{z}\right)=0$, and green dots (right column) evanescent states with $\Re\left(k_{z}\right)=0.5$ i.u.

constants are indeed very similar at the three energies. The only exception from this are the very small circles at $\bar{\Gamma}$ in Fig. 3(a) and $\bar{M}$ in Fig. 3(c), indicating the contribution from the very flat bands at the band edges. However, these contributions can only be seen very close to the energies corresponding to the conduction-band edge [Fig. 3(a)] and the valence-band edge [Fig. 3(c)]. Furthermore, one might notice the crosslike structures reflecting the typical flat bands of a perovskite structure.

As a second example of a ferroelectric insulator, which might potentially be relevant for a ferroelectric barrier material, we discuss $\mathrm{PbTiO}_{3}$. In this case, we used the experimentally determined structure with atomic positions as specified in Table I.

The complex band structure depicted in Fig. 4 shows some similarities with that of $\mathrm{BaTiO}_{3}$ discussed so far. In particular, the coexistence of both strongly dispersing bands as well as of bands with very little dispersion can be observed again. Also, the presence of the ferroelectric distortion manifests itself by a loss of degeneracy at the high-symmetry points. The band gap is indirect, with the conduction-band minimum formed by an extremely flat band along $\Gamma-X$ and the top of the valence band at the $X$ point. In our calculation, the band gap is about $1.3 \mathrm{eV}$.

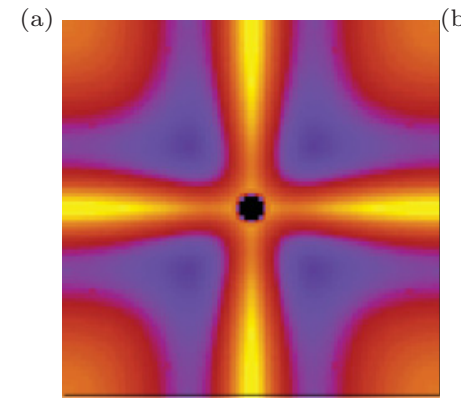

(c)

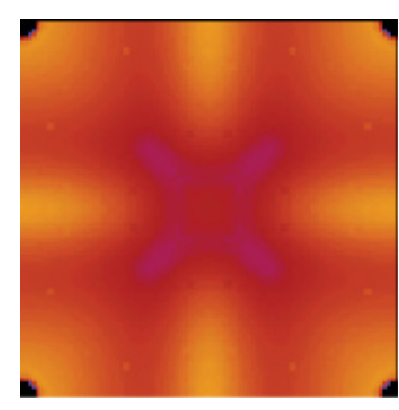

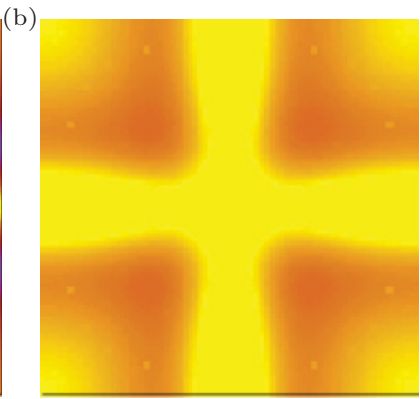

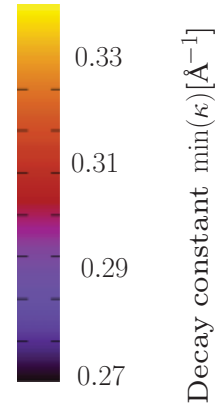

FIG. 3. (Color online) Minimal $\operatorname{Im}\left(k_{z}\right)$ in ferroelectric $\mathrm{BaTiO}_{3}$ for the whole two-dimensional Brillouin zone. The three panels (a), (b), and (c) correspond to energies of $\varepsilon=1.84,0.91$, and $0.00 \mathrm{eV}$, respectively. The black spots in the panels (a) and (c) show the areas in which the Bloch states at the band-gap edges enter.

However, in several aspects, the complex band structure of $\mathrm{PbTiO}_{3}$ and the expected decay of the wave functions is more complicated than that of $\mathrm{BaTiO}_{3}$. At energies only slightly above the valence band, one would expect significant tunneling due to the evanescent state emerging from the maximum of the valence band at the $X$ point. At higher energies, however, many bands with very similar imaginary part of the $k$ vector, i.e., with very similar decay, can be found. For electrons with normal incidence, corresponding to the uppermost panel of Fig. 4, the band emerging from the real band at $\Gamma$ again is rather similar to the corresponding states in $\mathrm{BaTiO}_{3}$, but there is also a slowly decaying state forming a loop from $2.2 \mathrm{eV}$ down to $-1.0 \mathrm{eV}$ emerging from the $X$ point. These evanescent states will also have a real part $\operatorname{Re}\left(k_{z}\right)=0.5$ i.u. The two evanescent states in the lowest panel of Fig. 4 also have a small decay constant in a large energy range of the band gap and, hence, can be relevant for tunneling. A common feature of all these bands is their finite decay at the conduction-band edge. The flat conduction band has such a high effective mass that it can not contribute to any tunneling as the corresponding evanescent states acquire a huge decay constant even at energies just below the conduction-band edge. Hence, many more bands of different character possibly contribute to tunneling in $\mathrm{PbTiO}_{3}$, with their actual significance for tunneling in an actual junction

TABLE I. Lattice parameters of $\mathrm{PbTiO}_{3}$ (from Ref. 28).

\begin{tabular}{ll}
\hline In-plane lattice constant & $3.895 \AA$ \\
Perpendicular lattice constant & $4.171 \AA$ \\
Displacement of Ti & $0.023 \AA$ \\
Displacement of O (Ti plane) & $0.062 \AA$ \\
Displacement of O (Pb plane) & $0.064 \AA$ \\
\hline \hline
\end{tabular}




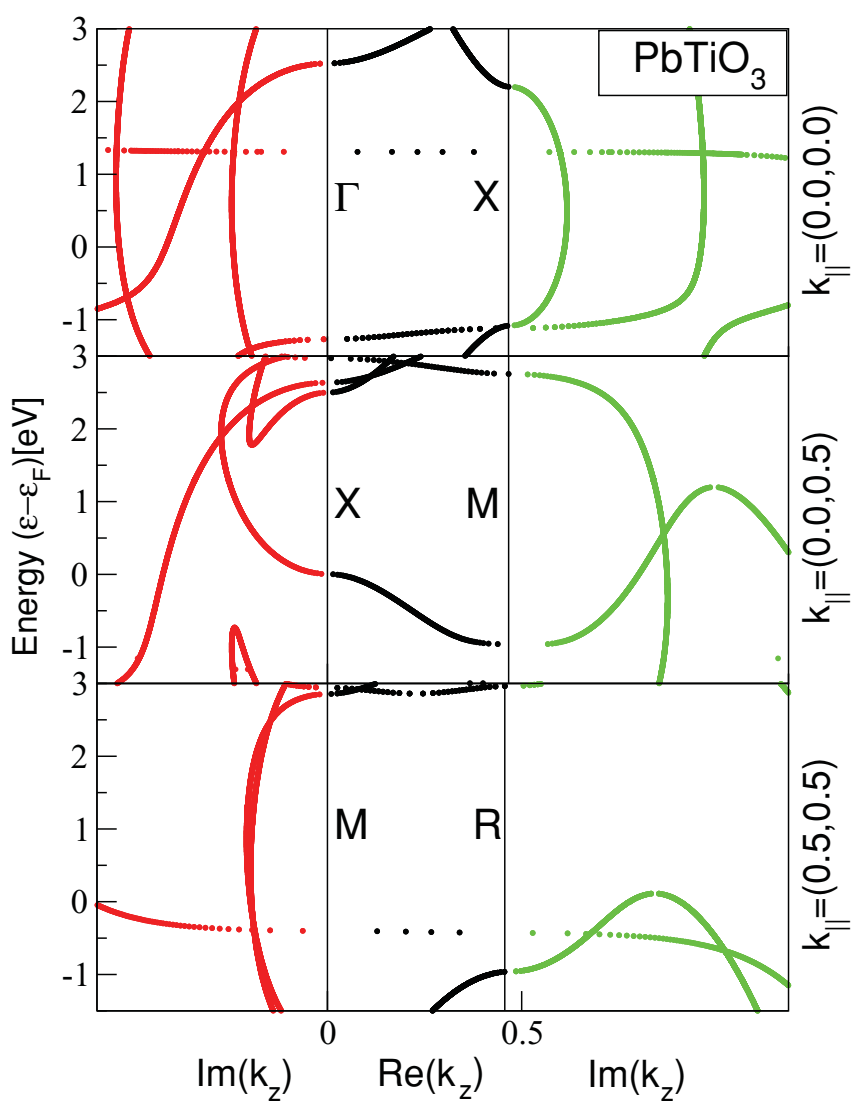

FIG. 4. (Color online) Complex band structure of $\mathrm{PbTiO}_{3}$. See caption of Fig. 2 for details.

depending on the interface and the electronic structure of the attached lead materials. However, the electronic tunneling through these states can not be expected to be described by the effective mass model; this model may be only applicable to the tunneling of holes with small energy.
The main origin of the little variation of the decay with the energy in the band gaps of $\mathrm{BaTiO}_{3}$ and $\mathrm{PbTiO}_{3}$ is the tetragonal distortion accompanying the ferroelectric phase. In a tight-binding picture, this distortion breaks the symmetry between the states with large hopping across the barrier and states with hopping within planes parallel to the barrier. As the conduction- and valence-band edges are formed by states orientated parallel to the interface, these states show little dispersion in the $k_{z}$ direction normal to the interface and these flat bands do not contribute to the tunneling.

\section{CONCLUSION}

The most basic origin of a tunneling electroresistance effect was discussed: the differences in the barrier height and shape and the corresponding variation of the decay of the wave function. While the simple description by an effective mass model reveals a TER in cases in which the barrier potential height is modified upon switching the polarization state, our investigation of the complex band structure of the two prototype ferroelectrics $\mathrm{BaTiO}_{3}$ and $\mathrm{PbTiO}_{3}$ indicated a highly nontrivial dependence of the decay of the tunneling wave function on the barrier potential. In $\mathrm{BaTiO}_{3}$, little variation of the decay is expected, resulting in a very low TER. Hence, despite its simplicity, $\mathrm{BaTiO}_{3}$ might not be the optimal choice as a barrier material. It should be stressed, however, that our results only describe the TER due to the intrinsic electronic structure of the barrier. Interface effects can still give a TER in $\mathrm{BaTiO}_{3}$-based junctions, which should be mostly independent of the barrier thickness. The more complex situation in $\mathrm{PbTiO}_{3}$ makes it even harder to predict the magnitude of the TER in this material. In conclusion, our results demonstrate that one should avoid simple interpretations of tunneling through ferroelectric barriers in terms of effective mass models or similar arguments, as these might be completely misleading.
${ }^{1}$ H. Kohlstedt, N. A. Pertsev, J. Rodríguez Contreras, and R. Waser, Phys. Rev. B 72, 125341 (2005).

${ }^{2}$ M. Y. Zhuravlev, R. F. Sabirianov, S. S. Jaswal, and E. Y. Tsymbal, Phys. Rev. Lett. 94, 246802 (2005).

${ }^{3}$ M. Bibes and A. Barthelemy, IEEE Trans. Electron Devices 54, 1003 (2007).

${ }^{4}$ A. Barthelemy, A. Fert, K. Bouzehouane, S. Fusil, M. Bibes, M. Gajek, and J. Fontcuberta, Nat. Mater. 6, 296 (2007).

${ }^{5}$ P. Maksymovych, S. Jesse, P. Yu, R. Ramesh, A. P. Baddorf, and S. V. Kalinin, Science 324, 1421 (2009).

${ }^{6}$ A. Gruverman, D. Wu, H. Lu, Y. Wang, H. W. Jang, C. M. Folkman, M. Y. Zhuravlev, D. Felker, M. Rzchowski, C.-B. Eom, and E. Y. Tsymbal, Nano Lett. 9, 3539 (2009).

${ }^{7}$ V. Garcia, S. Fusil, K. Bouzehouane, S. Enouz-Vedrenne, N. D. Mathur, A. Barthélémy, and M. Bibes, Nature (London) 460, 81 (2009).

${ }^{8}$ V. Garcia, M. Bibes, L. Bocher, S. Valencia, F. Kronast, A. Crassous, X. Moya, S. Enouz-Vedrenne, A. Gloter, D. Imhoff, C. Deranlot,
N. D. Mathur, S. Fusil, K. Bouzehouane, and A. Barthélémy, Science 327, 1106 (2010).

${ }^{9}$ E. Y. Tsymbal and H. Kohlstedt, Science 313, 181 (2006).

${ }^{10}$ M. Y. Zhuravlev, S. S. Jaswal, E. Y. Tsymbal, and R. F. Sabirianov, Appl. Phys. Lett. 87, 222114 (2005).

${ }^{11}$ J. P. Velev, C. G. Duan, K. D. Belashchenko, S. S. Jaswal, and E. Y. Tsymbal, Phys. Rev. Lett. 98, 137201 (2007).

${ }^{12}$ J. P. Velev, C.-G. Duan, J. D. Burton, A. Smogunov, M. K. Niranjan, E. Tosatti, S. S. Jaswal, and E. Y. Tsymbal, Nano Lett. 9, 427 (2009).

${ }^{13}$ M. Y. Zhuravlev, Y. Wang, S. Maekawa, and E. Y. Tsymbal, Appl. Phys. Lett. 95, 052902 (2009).

${ }^{14}$ N. F. Hinsche, M. Fechner, P. Bose, S. Ostanin, J. Henk, I. Mertig, and P. Zahn Phys. Rev. B 82, 214110 (2010).

${ }^{15} \mathrm{~J}$. Wang, S. Ju, and Z. Y. Li, J. Phys. D 43, 135003 (2010).

${ }^{16}$ Y. Zheng and C. H. Woo, Nanotechnology 20, 075401 (2009). 
${ }^{17}$ X. Luo, S. P. Lin, B. Wang, and Y. Zheng, Appl. Phys. Lett. 97, 012905 (2010).

${ }^{18}$ R. Landauer, IBM J. Res. Dev. 1, 223 (1957).

${ }^{19}$ V. Heine, Surf. Sci. 2, 1 (1964).

${ }^{20}$ E. Krasovskii, Solid State Commun. 93, 775 (1995).

${ }^{21}$ Y.-C. Chang and J. N. Schulman, Phys. Rev. B 25, 3975 (1982).

${ }^{22}$ G. Wachutka, Phys. Rev. B 34, 8512 (1986).

${ }^{23}$ D. Wortmann, H. Ishida, and S. Blügel, Phys. Rev. B 65, 165103 (2002).

${ }^{24}$ [http://www.flapw.de].
${ }^{25}$ J. P. Perdew, J. A. Chevary, S. H. Vosko, K. A. Jackson, M. R. Pederson, D. J. Singh, and C. Fiolhais, Phys. Rev. B 46, 6671 (1992).

${ }^{26}$ J. P. Perdew, K. Burke, and M. Ernzerhof, Phys. Rev. Lett. 77, 3865 (1996).

${ }^{27}$ F. Freimuth, Y. Mokrousov, D. Wortmann, S. Heinze, and S. Blügel, Phys. Rev. B 78, 35120 (2008).

${ }^{28}$ M. Kupriyanov, D. Kovtun, A. Zakharov, G. Kushlyan, S. Yagunov, R. Kolesova, and K. Abdulvakhidov, Phase Transitions 64, 145 (1998). 